\title{
MicroRNA-34a suppresses colorectal cancer metastasis by regulating Notch signaling
}

\author{
XUEMEI ZHANG ${ }^{1-3^{*}}$, FEIYAN AI ${ }^{1,2^{*}}$, XIAYU LI ${ }^{1,2}$, LI TIAN $^{1,2}$, XIAOYAN WANG ${ }^{1,2}$, \\ SHOURONG SHEN ${ }^{1,2}$ and FEN LIU ${ }^{1,2}$ \\ ${ }^{1}$ Department of Gastroenterology, The Third Xiangya Hospital of Central South University, Changsha, Hunan 410013; \\ ${ }^{2}$ Hunan Key Laboratory of Nonresolving Inflammation and Cancer, Changsha, Hunan 410008; \\ ${ }^{3}$ Cancer Research Institute, Central South University, Changsha, Hunan 410013; ${ }^{4}$ Key Laboratory of Carcinogenesis \\ and Cancer Invasion, Ministry of Education, Changsha, Hunan 410008, P.R. China
}

Received September 10, 2015; Accepted April 4, 2017

DOI: $10.3892 / \mathrm{ol} .2017 .6444$

\begin{abstract}
Dysregulation of microRNA (miRNA/miR) expression is causally associated with cancer initiation and progression. However, the precise mechanisms by which dysregulated miRNAs induce colorectal tumorigenesis remain unknown. In the present study, downregulation of miR-34a was identified in colorectal cancer cell lines and clinical specimens. Clinical studies revealed that miR-34a expression was negatively associated with distant metastasis, and positively associated with differentiation and survival of human colorectal cancer specimens. In vitro miRNA functional assays demonstrated that miR-34a bound to the putative 3'-untranslated regions of Notch1 and Jagged1 in SW480 cells, and thereby attenuated the migration and invasion of the colon cancer cells. It was additionally identified that miR-34a downregulated the expression of vimentin and fibronectin via Notch1 and Jagged1. Overall, these data indicate that miR-34a serves a key role in suppressing colorectal cancer metastasis by targeting and regulating Notch signaling.
\end{abstract}

\section{Introduction}

Colorectal cancer (CRC) is the third most common type of cancer, and the leading cause of cancer-associated mortality worldwide (1). Previous studies suggest that $\sim 20 \%$ of the patients diagnosed with CRC exhibit metastatic disease, and an additional 30-35\% develop metastases later with tumor progression $(2,3)$. Despite significant therapeutic advances,

Correspondence to: Dr Fen Liu, Department of Gastroenterology, The Third Xiangya Hospital of Central South University, 138 Tong-Zi-Po Road, Changsha, Hunan 410013, P.R. China E-mail: liufencsu@163.com

${ }^{*}$ Contributed equally

Key words: microRNAs, colorectal cancer, Notch1, Jagged1, cell metastasis $\sim 60 \%$ of all patients receiving curative resection will experience local recurrence or distant metastases (4). Therefore, a comprehensive understanding of the molecular mechanisms associated with CRC metastasis is imperative to facilitate early diagnosis in high-risk populations.

In previous years, rapid advances have occurred in the use of microRNAs (miRNAs/miRs) as novel biomarkers in patients with cancer. miRNAs are short non-coding RNA molecules, which have been widely identified to be involved in malignant transformation by inhibiting the expression of target genes through translational inhibition or transcriptional silencing (5). In cancer, miRNAs may act as either oncogenes or tumor suppressor genes that affect almost every basic cellular process. However, the aberrant expression and potential role of miRNAs in CRC remains largely unclear, particularly in the metastatic process (6).

Of note, the dysregulation of the members of miR-34 family in various types of cancer has been associated with tumorigenesis, growth and progression $(7,8)$. For instance, miR-34a as a tumor suppressor has been identified in solid tumors including lung cancer (9), multiple myeloma (10), neuroblastoma $(11,12)$, glioblastoma $(13,14)$, cervical carcinoma (15), breast cancer (16) and colon cancer (17). miR-34a promotes epithelial-to-mesenchymal transition (EMT)-mediated invasion and metastasis via feedback and feedforward loops with other miRNAs and proteins (18-20). In addition, it has been suggested that miR-34a mimics were a novel potential therapeutic agent targeting multiple myeloma and pancreatic cancer $(10,21)$. The tumor suppressor miR-34a is a cell-fate determinant in early-stage dividing colon cancer stem cells (22-24). It is therefore critical to investigate the regulatory function of miR-34a in cancer, including CRC.

In the present study, miR-34a expression was determined in colon cancer cell lines and clinical CRC samples. Functional studies on miR-34a demonstrated that it disrupted colon cancer cell growth and invasion potentially via inhibition of Notch1 and Jagged1 expression. Vimentin and fibronectin function as the downstream molecules of these pathways, contributing to progression of CRC. These studies provide novel insights into the underlying molecular mechanisms of CRC cell metastasis, highlighting the unique regulatory role of miRNAs. 


\section{Materials and methods}

Cell lines and culture. Human colon cancer cell lines SW480, SW620, HT29, and the human embryonic kidney HEK293 cell line were purchased from the Cell Bank of the Chinese Academy of Sciences (Shanghai, China) and grown in RPMI-1640 medium supplemented with $10 \%$ fetal bovine serum (FBS) (both from Hyclone, GE Healthcare Life Sciences, Logan, UT, USA), $100 \mathrm{U} / \mathrm{ml}$ penicillin $\mathrm{G}$ and $100 \mathrm{mg} / \mathrm{ml}$ streptomycin (both from Beyotime Institute of Biotechnology, Shanghai, China). The human colonic epithelial cell line NCM460 was purchased from INCELL Corporation LLC (San Antonio, TX, USA) and grown in M3:10 medium (INCELL Corporation LLC). The cells were cultured at $37^{\circ} \mathrm{C}$ in a $5 \% \mathrm{CO}_{2}$ incubator.

Plasmids and reagents. Full-length Notch1 and Jagged1 cDNA lacking the 3'-untranslated region (UTR) were purchased from GeneCopoeia (Rockville, MD, USA) and subcloned into the eukaryotic expression vector pIRES (Clontech Laboratories, Inc., Mountain View, CA, USA). The empty pIRES vector was used as a negative control. Rabbit monoclonal anti-Notch1 (cat no. EP1238Y) and anti-Jagged1 (cat no. EPR4290) were purchased from Epitomics (Abcam, Cambridge, UK), mouse monoclonal anti-GAPDH (cat no. MAB374) was purchased from EMD Millipore (Billerica, MA, USA), and mouse monoclonal anti-vimentin (cat no. sc-6260), anti-fibronectin (cat no. $\mathrm{C} 6 \mathrm{~F} 10$ ) and horseradish peroxidase (HRP)-conjugated goat anti-rabbit IgG (cat no.sc-2004) antibodies were purchased from Santa Cruz Biotechnology, Inc. (Dallas, TX, USA). Synthetic miR-34a mimic and negative control (NC) were purchased from GenePharma Co., Ltd. (Shanghai, China). The miR-34a LNA 5'-digoxigenin (DIG)-labeled (cat no. 612537-340) and scrambled LNA 5'-DIG-labeled probes (cat no. 699004-340) were purchased from Exiqon A/S (Vedbaek, Denmark). The probe sequences are as follows: miR-34a, AGGGCAGTATAC TTGCTGAT; and scramble, GTGTAACACGTCTATACG CCCA. The probes were detected using an enhanced sensitive ISH detection kit (cat no. MK1030; Wuhan Boster Biological Technology, Ltd., Wuhan, China).

In situ hybridization (ISH). A tissue microarray (TMA) containing 103 samples of human CRC tissue specimens with their matched distant normal mucosa tissues $(>10 \mathrm{~cm}$ away from the primary tumor) was used in the present study. The high-density CRC tissue microarray from Chinese specimens collected in March 2006 from the Third Xiangya Hospital of Central South University (Changsha, China) was constructed by the study group. Written informed consent was obtained from patients. The present study was approved by the Ethical Review Committees of the Third Xiangya Hospital of Central South University, Hunan Key Laboratory of Nonresolving Inflammation and Cancer and the Central South University (Changsha, China). Histology of all slides was reviewed by two expert pathologists. The cancer stage was made according to the TNM classification criteria (25). The TMAs (4 $\mu \mathrm{m})$ were dewaxed and treated with $3 \% \mathrm{H}_{2} \mathrm{O}_{2}$ and proteinase $\mathrm{K}$ to inactivate endogenous peroxidases. The slides were treated with pepsin in $3 \%$ citric acid. The slides were hybridized at $56^{\circ} \mathrm{C}$ overnight with $50 \mathrm{nM}$ miR-34a LNA or scrambled LNA. Following washing 3 times with $2 \mathrm{X}$ saline-sodium citrate buffer, sections were blocked in blocking buffer (cat no. MK1030; Wuhan Boster Biological Technology, Ltd.) at $37^{\circ} \mathrm{C}$ for $30 \mathrm{~min}$ and then incubated with $1 \mathrm{X}$ anti-DIG Fab fragment antibody (cat no. MK1030; Wuhan Boster Biological Technology, Ltd.) at room temperature for $2 \mathrm{~h}$ for the hybridization reaction. The immunoreaction was detected using 3-diaminobenzidine tetrahydrochloride (cat no. 0031; Fuzhou Maixin Biotech Co., Ltd., Fuzhou, China). The sections were counterstained with $5 \mathrm{~g} / \mathrm{l}$ hematoxylin at room temperature for $3 \mathrm{~min}$. Following dehydration and mounting, the sections were observed under an inverted microscope (Nikon Corporation, Tokyo, Japan) with magnifications of $\mathrm{x} 100$ or $\mathrm{x} 200$. The staining intensity of miR-34a was scored as: $0-1$, negative; 1-2, weak; 2-3, medium; and 3-4, strong. The percentages of miR-34a cells in 3 representative high-power fields of individual samples were determined. The miR-34a expression was calculated in terms of the product of the intensity scores and the percentage of positive cells. Individual samples were evaluated by at least 2 blinded pathologists. Scores $\geq 2$ were used to define specimens with a high expression, and a score of $<2$ indicated low expression.

miRNA target prediction. Potential target genes of miR-34a were predicted and analyzed using 4 web-based bioinformatics tools: TargetScan, miRWalk, microRNA.org and RNA22. The number of false positive predictions was markedly decreased by selecting the putative target genes that were predicted by $\geq 2$ programs.

Transient transfection of miRNA precursors. The SW480 cells were seeded into 6-well plates. The cells were transfected by nucleofection with $100 \mathrm{nM}$ miR-34a mimic or mimic NC using HiPerFect Transfection reagent (Qiagen, Inc., Valencia, CA, USA), according to the manufacturer's protocol. At $48 \mathrm{~h}$ post-transfection, the reverse transcription-quantitative polymerase chain reaction (RT-qPCR) was performed to verify the transfection efficiency.

$R T-q P C R$. Total RNA from cells and tissues was extracted using TRIzol reagent (Invitrogen; Thermo Fisher Scientific, Inc., Waltham, MA, USA). The Hairpin-it ${ }^{\mathrm{TM}}$ MicroRNA Quantitation PCR kit was purchased from GenePharma Co., Ltd. The RT-qPCR was performed according to the manufacturer's protocol. At least three biological replicates were performed for each case. The qPCR analysis was performed using the iQ5 Real-Time PCR system (Bio-Rad Laboratories, Inc., Hercules, CA, USA). The miRNA PCR quantification used the $2^{-\Delta \Delta C q}$ method (26) against U6 for normalization.

The expression of mRNA was evaluated using the SYBR Premix Ex Taq II (Tli RNaseH Plus) Real-Time qPCR kit (Takara Biotechnology Co., Ltd., Dalian, China). The mRNA PCR quantification used the $2^{-\Delta \Delta C q}$ method (26) against GAPDH for normalization. The RT-qPCR primers, including E-cadherin, vimentin, fibronectin, zinc finger protein SNAI2 (Slug), zinc finger protein SNAI1 (Snail), GAPDH and zinc finger E-box-binding homeobox 1 (ZEB1), are summarized in Table I.

Luciferase reporter assay. Human Notch1 (accession number NM_017617) and Jagged1 (NM_000214) 3'-untranslated 
Table I. Human primers for quantitative polymerase chain reaction.

\begin{tabular}{lll}
\hline Gene & \multicolumn{1}{c}{ Forward primer (5'-3') } & \multicolumn{1}{c}{ Reverse primer (5'-3') } \\
\hline Pre-microRNA-34a & UGGCAGUGUCUUAGCUGGUUGU & AACCAGCUAAGACACUGCCAUU \\
Notch1 & AGCCTCAACATCCCCTACAA & CCACGAAGAACAGAAGCACA \\
Jagged1 & ACGGGAAGTGCAAGAGTCAG & GTTTCACAGTAGGCCCCCTC \\
Epithelial cadherin & TTCTGGAAGGAATGGAGGAGTC & ACCTGGAATTGGGCAAATGTG \\
Vimentin & AGATGGCCCTTGACATTGAG & TGGAAGAGGCAGAGAAATCC \\
Fibronectin & GGTGACACTTATGAGCGTCCTAAA & AACATGTAACCACCAGTCTCATGTG \\
ZEB1 & GCACAACCAAGTGCAGAAGA & GCCTGGTTCAGGAGAAGATG \\
Snail & GCTGCAGGACTCTAATCCAGAGTT & GACAGAGTCCCAGATGAGCATTG \\
Slug & AGATGCATATTCGGACCCAC & CCTCATGTTTGTGCAGGAGA \\
GAPDH & AACGGATTTGGTCGTATTGG & TTGATTTTGGAGGGATCTCG
\end{tabular}

Slug, zinc finger protein SNAI2; Snai1, zinc finger protein SNAI1; ZEB1, zinc finger E-box-binding homeobox 1.

Table II. Primers and oligonucleotides.

Forward primer $\left(5^{\prime}-3^{\prime}\right)$

3'UTR of wild 1 Notch 1

3'UTR of mutant1 Notch1

3'UTR of wild2 Notch1

3'UTR of mutant12 Notch1

3'UTR of wild 1 Jagged1

3'UTR of mutant1 Jagged1

3'UTR of wild2 Jagged1

3'UTR of mutant2 Jagged1

CGCGTTTTATTTATGTACTTTTATTT
TACACAGAAACACTGCCTTTTTATT
TATATGTAA

CGCGTTTTATTTATGTACTTTT ATTTTACACAGAAA $\underline{A A A A T T T T T T}$ TATTTATATGTAA

CGCGTTACCCTTTTCTGGGGAA AGACACTGCCTGGGCTGACCCCGGT GGCGGCCCCAGCA

CGCGTTACCCTTTTCTGGGGAAA GAAAAATTTTGGGCTGACCCCGGT GGCGGCCCCAGCA

CGCGTACCGCGGGCACTGCCGCCG CTAGGTAGAGTCTGAGGGCTTGTAG TTCTTTAAA

CGCGTACCGCGGGAAAATTTGC CGCTAGGTAGAGTCTGAGGGCTTGT AGTTCTTTAAA

CGCGTTTTAGATTTGCCATAGAGTA CACTGCCTGCCTTAAGTGAGGAA ATCAAAGA CGCGTTTTAGATTTGCCATAGAGTA AAAATTTTGCCTTAAGTGAGG AAATCAAAGA
Reverse primer (5'-3')

AGCTTTACATATAAATAAAAAGG
CAGTGTTTCTGTGTAAAATAAA
AGTACATAAATAAAA
AGCTTTACATATAAATAAAAAA
AATTTTTTCTGTGTAAAATAAAAG
TACATAAATAAAA
AGCTTGCTGGGGCCGCCACCG
GGGTCAGCCCAGGCAGTGTCTTTC
CCCAGAAAAGGGTAA
AGCTTGCTGGGGCCGCCACCG
GGGTCAGCCCAAAATTTTTCTTTCC
CCAGAAAAGGGTAA
AGCTTTTAAAGAACTACAAGC
CCTCAGACTCTACCTAGCG
GCGGCAGTGCCCGCGGTA
AGCTTTTAAAGAACTACAAGC
CCTCAGACTCTACCTAGCGGC
AAATTTCCCGCGGTA
AGCTTCTTTGATTTCCTCACTTAA
GGCAGGCAGTGTACTCTATG
GCAAATCTAAAA
AGCTTCTTTGATTTCCTCACTTAA
GGCAAAATTTTACTCTATG
GCAAATCTAAAA

The underlined sections of the sequences are mutation sites. UTR, untranslated region.

regions (UTRs) are predicted to contain two putative binding sites of miR-34a. Notch1 and Jagged1 wild-type (WT) 3'-UTRs and mutant 3'-UTRs with 6 base mutations at putative seed regions were synthesized and cloned into the pIRES vector with the restriction sites for HindIII and SpeI at the two ends of the oligonucleotides. The WT and mutant oligonucleotide sequences used are summarized in Table II. In total, 9 luciferase reporters (LR) were constructed and referred to as LR-blank (no insertion), LR-34a/Notch1w1 (where w1 indicates WT site 1), LR-34a/Notch1w2 (where w2 indicates WT site 2), LR-34a/Notch1m1 (where $\mathrm{m} 1$ indicates site 1 mutant), LR-34a/Notch1 $\mathrm{m} 2$ (where $\mathrm{m} 2$ indicates site 2 mutant), LR-34a/ Jagged1w1, LR-34a/Jagged1w2, LR-34a/ Jagged1m1 and LR-34a/Jagged1m2. HEK293 cells were cultured in RPMI-1640 medium supplemented with 10\% FBS in 24-well plates at $37^{\circ} \mathrm{C}$ overnight, and then co-transfected with $100 \mathrm{ng}$ 
of one of the reporter plasmids and 10 pmol miR-34a mimic or mimic NC using MegaTran 1.0 Transfection reagent (OriGene Technologies, Inc., Rockville, MD, USA). At $24 \mathrm{~h}$ post-transfection, firefly and Renilla luciferase activities were consecutively measured using the commercial dual-luciferase reporter system (Promega Corporation, Madison, WI, USA). The Renilla luciferase signal was normalized to the firefly luciferase signal for each individual analysis.

Western blot analysis. Cells were lysed in radioimmunoprecipitation assay buffer containing $1 \%$ protease inhibitor cocktail (Pierce; Thermo Fisher Scientific, Inc.). The protein concentrations in the lysates were measured using a bicinchoninic acid protein assay kit (Pierce; Thermo Fisher Scientific, Inc.). Total protein $(50 \mu \mathrm{g} /$ lane) was separated on a Bio-Rad Bis-Tris Gel system (Bio-Rad Laboratories, Inc.) and electrotransferred onto polyvinylidene fluoride membranes. Subsequent to blocking with $5 \%$ bovine serum albumin (cat no. SW3015; Beijing Solarbio Science \& Technology Co.,Ltd., Beijing, China) at room temperature for $1 \mathrm{~h}$, the membranes were serially incubated with anti-Notch1 (1:500 dilution), anti-Jagged1, anti-vimentin (1:200 dilution), anti-fibronectin (1:500 dilution) and anti-GAPDH $(1: 1,000)$ primary antibodies at $4{ }^{\circ} \mathrm{C}$ overnight, followed by incubation with the HRP-conjugated goat anti-rabbit IgG secondary antibody (1:1,000 dilution) at $37^{\circ} \mathrm{C}$ for $2 \mathrm{~h}$. Chemiluminescence using Luminata Forte Western horseradish peroxidase substrate (Merck KGaA, Darmstadt, Germany) was visualized using a ChemiDoc XRS+ system (Bio-Rad Laboratories, Inc.). The densities of the bands were quantified using Image Lab 2.0 software (Bio-Rad Laboratories, Inc.).

Wound closure assay. SW480 cells $\left(1 \times 10^{6}\right.$ cells/well) were cultured overnight at $37^{\circ} \mathrm{C}$. SW480 cells were transfected with miR-34a (or mimic NC) followed by Notch1 or Jagged1 transfection. Following the cells in the 6-well dish attaining $90 \%$ confluence, a wound was created using a $10 \mu l$ pipette tip. Following washing 3 times in PBS to remove cellular debris, cells were cultured in RPMI-1640 medium with $2 \%$ serum. Cell migration at the wound sites was documented using an inverted microscope (Nikon Corporation) with a magnification of $\times 200$ at the indicated time-points ( 0 and 48 h). Cells in 3 randomly selected areas were counted.

Invasion assay. Corning Costar Transwell 24-well plates (8- $\mu \mathrm{m}$ pores; Corning Incorporated, Corning, NY, USA) coated with BD Matrigel matrix (BD Biosciences, Franklin Lakes, NJ, USA) were maintained for $1 \mathrm{~h}$ at $37^{\circ} \mathrm{C}$, followed by the addition of $1 \times 10^{5}$ transfected cells suspended in $200 \mu \mathrm{l}$ medium with $1 \%$ serum into the top of each well insert. Normal growth medium was added to the bottom wells. The cells were allowed to migrate for $24 \mathrm{~h}$ at $37^{\circ} \mathrm{C}$. The migrated cells were fixed with $10 \%$ methanol for $15 \mathrm{~min}$. The invading cells on the lower surface of the membrane were stained with $0.5 \%$ crystal violet for $5 \mathrm{~min}$ at room temperature. The stained cells were counted under a microscope (Nikon Corporation). To minimize the bias, $\leq 5$ randomly selected fields at x100 magnification were counted, and the average number was calculated.

Statistical analysis. Statistical analysis was performed using the SPSS for Windows (version 17.0; SPSS, Inc., Chicago, IL,
USA) and GraphPad Prism (version 5; GraphPad Software, Inc., La Jolla, CA, USA). $\chi^{2}$ tests were used for categorical variables, including categorized miR-34a expression levels, clinical stage, histological differentiation, sex, age and metastasis of the colorectal tumors. Independent unpaired t-tests and one-way analysis of variance with Bonferroni post hoc testing were performed for the analysis of continuous variables in categories. Survival analysis was determined using the Kaplan-Meier estimator method. $\mathrm{P}<0.05$ was considered to indicate a statistically significant difference.

\section{Results}

Global repression of $m i R-34$ a expression. RT-qPCR was used to detect the expression of miR-34a in colon cancer cell lines SW480, SW620 and HT29, and normal colonic epithelial cell line NCM460. Compared with the normal colonic epithelial cells, miR-34a was identified to be downregulated $\sim 2.5$-fold in SW480 cells, 3-fold in HT29 cells and 6-fold in SW620 cells (Fig. 1A). In addition, compared with SW480, the relatively high metastatic SW620 and HT29 cells exhibited significantly decreased miR-34a expression, particularly in SW620 cells $(\mathrm{P}=0.014)$.

To verify the altered expression of miR-34a in human colorectal carcinogenesis, ISH was first conducted to evaluate miR-34a expression levels in 103 pairs of CRC tissues and their matched adjacent normal tissues using a TMA (representative images are presented in Fig. 1B). It was identified that miR-34a was apparently downregulated in the colorectal tumors compared with the normal tissues $(\mathrm{P}=0.000$; Table III). Next, the potential clinicopathological implications of altered miR-34a expression were assessed. Clinical samples were divided into low- and high-expression groups based on the miR-34a expression scores $>2$ or $<2$. Of the 103 normal colorectal samples, $88(85 \%)$ exhibited increased expression of miR-34a (Table III). Thus, miR-34a is underexpressed in CRC tissue compared with the normal colorectal mucosa (representative images are presented in Fig. 1C). In the 103 individuals with CRC, the miR-34a level was inversely associated with distant metastasis, and positively associated with differentiation and age $(\mathrm{P}=0.045,0.010$ and 0.020 , respectively; Table III). However, no association between miR-34a expression and sex and TNM stage was observed in patients with CRC. To additionally assess the significance of miR-34a in terms of clinical prognosis, a Kaplan-Meier estimator survival analysis was conducted using overall survival (OS). The results demonstrated that patients with a diminished miR-34a expression exhibited shorter OS times compared with patients expressing high miR-34a levels ( $\mathrm{P}=0.029$; Fig. 1D). These data demonstrated overt downregulation of miR-34a in CRC, suggesting that the expression levels of miR-34a was associated with the metastatic potential of CRC.

miR-34a directly targets and inhibits Notch1 and Jaggedl. The biological function of miR-34a was explored using several bioinformatics algorithms in order to identify potential miR-34a target genes. Among the candidate target genes, Notch1 and its ligand Jagged1 were identified. As presented in Fig. 2A, 2 miR-34a-binding sites were identified in the 3'-UTRs of Notch1 and Jagged1 mRNAs, with a perfect base 
Table III. Association of clinical and pathological features with miR-34a expression.

\begin{tabular}{|c|c|c|c|c|}
\hline \multirow[b]{2}{*}{ Variable } & \multirow[b]{2}{*}{ Cases (n) } & \multicolumn{2}{|c|}{ Expression of microRNA-34a } & \multirow[b]{2}{*}{ P-value } \\
\hline & & Low, n (\%) & High, n (\%) & \\
\hline Histological type & & & & 0.000 \\
\hline Normal tissues & 103 & $15(14.6)$ & $88(85.4)$ & \\
\hline Colorectal cancer tissues & 103 & $54(52.4)$ & $49(47.6)$ & \\
\hline Age, years & & & & 0.020 \\
\hline$\leq 56$ & 52 & $39(75.0)$ & $13(25.0)$ & \\
\hline$>56$ & 51 & $27(52.9)$ & $24(47.1)$ & \\
\hline Sex & & & & 0.182 \\
\hline Male & 59 & $34(57.6)$ & $25(42.4)$ & \\
\hline Female & 44 & $31(70.5)$ & $13(29.5)$ & \\
\hline Distant metastasis & & & & 0.045 \\
\hline No & 78 & $32(41.0)$ & $46(59.0)$ & \\
\hline Yes & 25 & $16(64.0)$ & $9(36.0)$ & \\
\hline Distant differentiation & & & & 0.010 \\
\hline High/moderate & 88 & $41(46.6)$ & $47(53.4)$ & \\
\hline Low & 15 & $13(86.7)$ & $2(13.3)$ & \\
\hline TNM stage & & & & 0.469 \\
\hline I-II & 48 & $29(60.4)$ & $19(39.6)$ & \\
\hline III-VI & 55 & $37(67.3)$ & $18(32.7)$ & \\
\hline
\end{tabular}

TNM, tumor-node-metastasis.
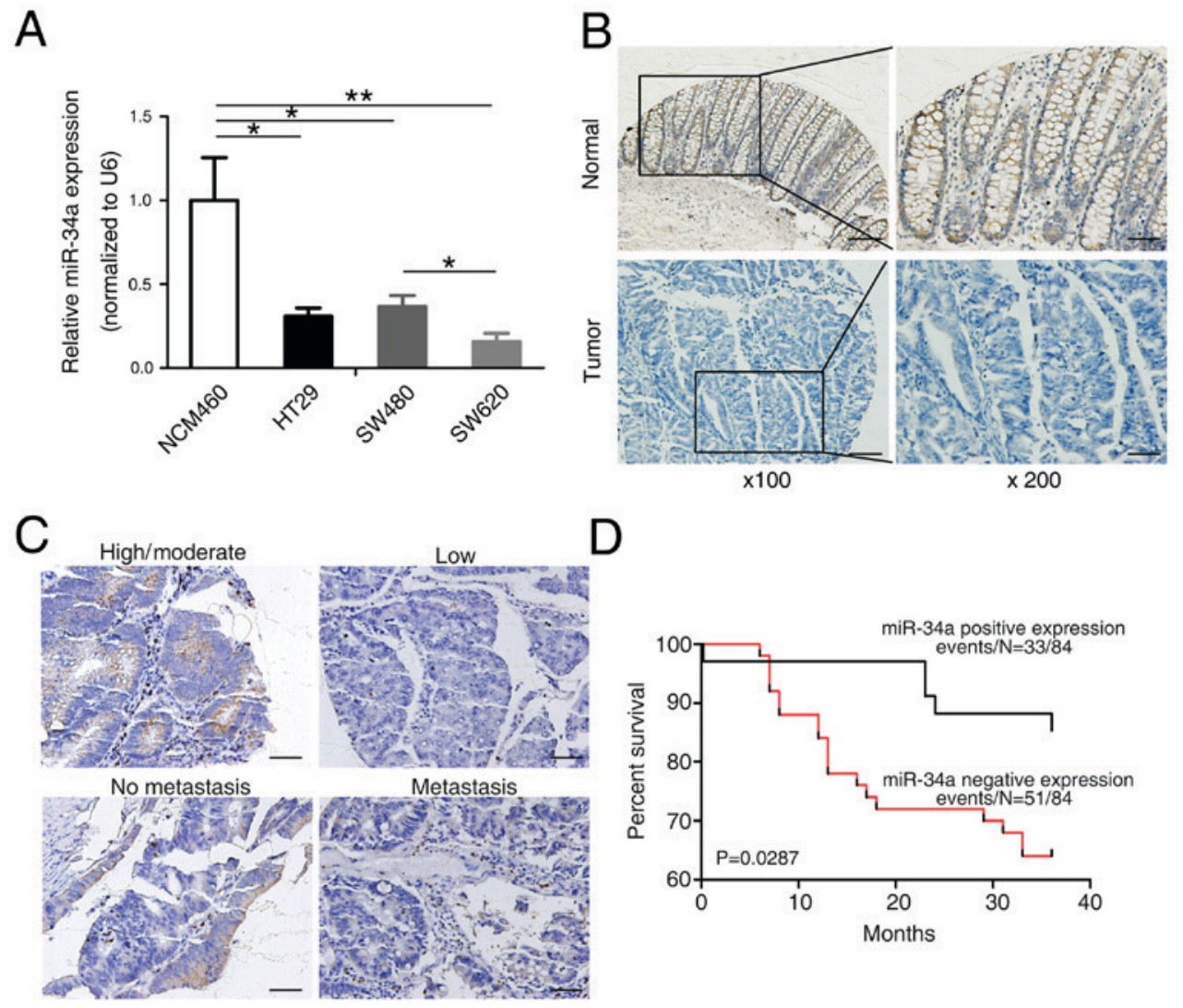

Figure 1. Expression of mature miR-34a in colorectal cancer cells and tissues. (A) Reverse transcription-quantitative polymerase chain reaction analysis of miR-34a expression in the potentially invasive colon cancer cell lines HT29, SW480 and SW620, and the normal colonic epithelial cell line NCM460. The data are expressed as the mean \pm standard deviation $(\mathrm{n}=3)$. ${ }^{*} \mathrm{P}<0.05$ and ${ }^{* *} \mathrm{P}<0.01$ vs. NCM460. (B) Colorectal cancer tissue microarray composed of normal colon tissue and colon adenocarcinoma tissue from patients was analyzed using in situ hybridization. Representative images of miR-34a expression (left panel: Original magnification, x100; scale bar, $100 \mu \mathrm{m}$; right panel: Original magnification, x200; scale bar, $50 \mu \mathrm{m})(\mathrm{C})$ Representative images of miR-34a expression in well-differentiated (high/moderate differentiation) colorectal cancer, poorly differentiated (low differentiation) colorectal cancer, non-metastatic tissues and metastasis tissues. Original magnification, x200; scale bar, $50 \mu \mathrm{m}$. (D) Kaplan-Meier estimator survival curves for patients according to the expression levels of miR-34a in tumors. miR, microRNA. 
A

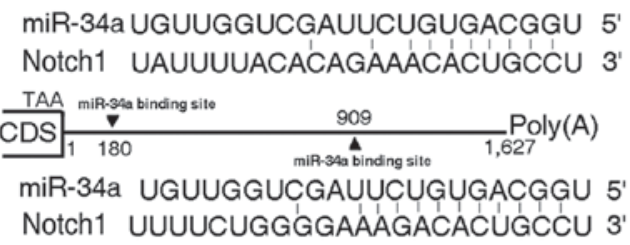

B
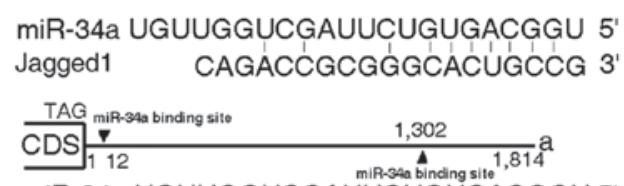

miR-34a UGUUGGUCGAUUCUUGUGACGGU $5^{\prime}$ Jagged1 AUUUGCCAUAGAGUACACUGCCU 3'
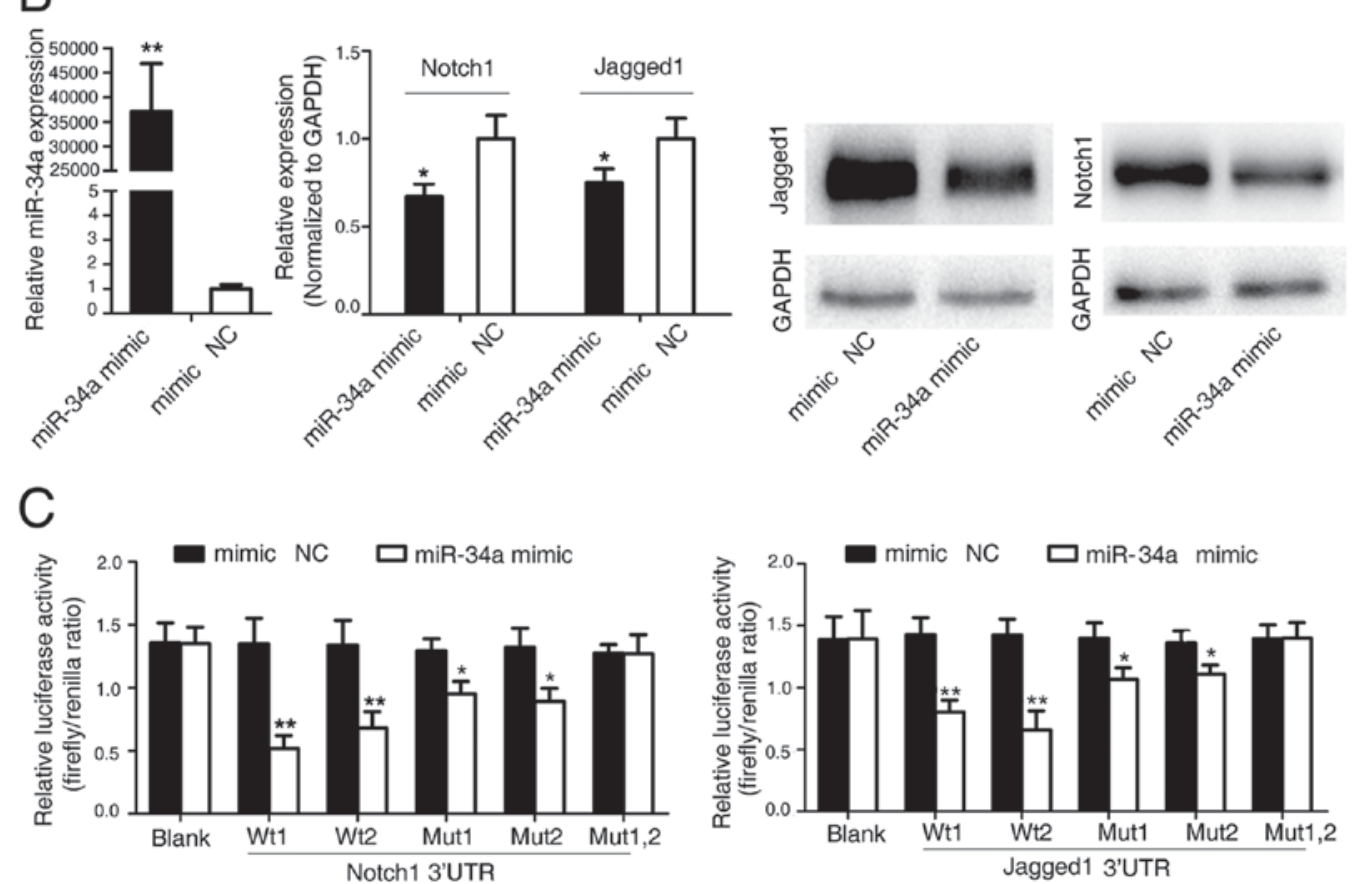

Figure 2. Notch1 and Jagged1 are direct target genes of human miR-34a. (A) Schematic representation of the predicted binding sites of miR-34a in the Notch1 and Jagged1 3' untranslated regions, and the sequence of the intact (wild-type) miR-34a and their mutant binding sites within the luciferase reporter vector. (B) miR-34a suppresses the expression of Notch1 and Jagged. Reverse transcription-quantitative polymerase chain reaction (middle panel) and Western blot analyses (right panel) of Notch1 and Jagged1 were performed following transfection of cells with an miR-34a mimic or mimic negative control (NC) for $48 \mathrm{~h}$ (left panel). The data are expressed as the mean $\pm \mathrm{SD}(\mathrm{n}=3)$. ${ }^{*} \mathrm{P}<0.05$ and ${ }^{* *} \mathrm{P}<0.01$ vs. mimic NC. (C) A total of 9 luciferase reporter constructs were created for the luciferase activity assay, namely LR-blank (no insertion), LR-34a/Noth1w1, LR-34a/Noth1w2, LR-34a/Jagged1w1, LR-34a/Jagged1w2, LR-34a/Noth1m1, LR-34a/Noth1m2, LR-34a/Jagged1m2 and LR-34a/Jagged1m2. Each of the constructs was co-transfected with an miR-34a mimic (or mimic NC) into HEK293 cells. Luciferase activities were measured $36 \mathrm{~h}$ post-transfection. The data are expressed as the mean $\pm \mathrm{SD}\left(\mathrm{n}=3\right.$ ). ${ }^{*} \mathrm{P}<0.05$ and ${ }^{* * *} \mathrm{P}<0.01 \mathrm{vs}$. mimic $\mathrm{NC}$. miR, microRNA; CDS, coding sequence; SD, standard deviation; NC, negative control; w1/2, wild-type 1/2; m1/m2, mutant 1/2.

pairing between the seed sequence of mature miR-34a and the miRNA-recognition element in the 3'-UTRs. A number of cellular functions and biochemical processes associated with tumorigenesis are modulated by Notch signaling, including EMT, proliferation, apoptosis, adhesion and angiogenesis (27). A previous study has identified Notch1 overexpression in various solid tumors, including CRC (28). Previous studies have also suggested that miR-34a suppressed tumor invasion via the downregulation of Notch1 and Jagged1 in cervical carcinoma and choriocarcinoma cells (15). Therefore, an initial screen of the effects of miR-34a on Notch1 and Jagged1 expression was performed. Initially, miR-34a inhibition of the expression of Notch1 and Jagged1 was determined by transfecting miR-34a mimic into the SW480 cells. It was identified that the miR-34a mimic significantly decreased the mRNA and protein levels of Notch1 and Jagged1 compared with the mimic NC (Fig. 2B).

Whether Notch1 and Jagged1 were direct targets of miR-34a was investigated using a luciferase reporter assay to test the binding of miR-34a to the 3'-UTR of Notch1 and Jagged1. The Notch1 and Jagged1 3'-UTRs containing the binding sites for miR-34a were subcloned into a luciferase reporter vector. The addition of an miR-34a mimic significantly suppressed the luciferase activity of the Notch1 and Jagged1 3'-UTRs following co-transfection of the luciferase vector (WT, mutant or blank control) with the miR-34a mimic into SW480 cells (Fig. 2C). Thus, these results confirm that miR-34a directly recognizes the 3'-UTRs of the Notch1 and Jagged1 mRNAs, leading to mRNA degradation and translational inhibition.

miR-34a suppresses CRC metastasis by targeting Notch1 and Jaggedl. Considering the aforementioned data, the role of miR-34a in the human colon cancer SW480 cell line was next investigated. The effects of miR-34a were assessed using wound-healing assays and Matrigel invasion assays following transfection of the cells with miR-34a mimic or mimic NC. It was identified that overexpressed miR-34a in SW480 cells significantly attenuated cell migration and invasion. The re-expression of Notch1 or Jagged1 (lacking an endogenous 3'-UTR) prevented this inhibition, suggesting that miR-34a 

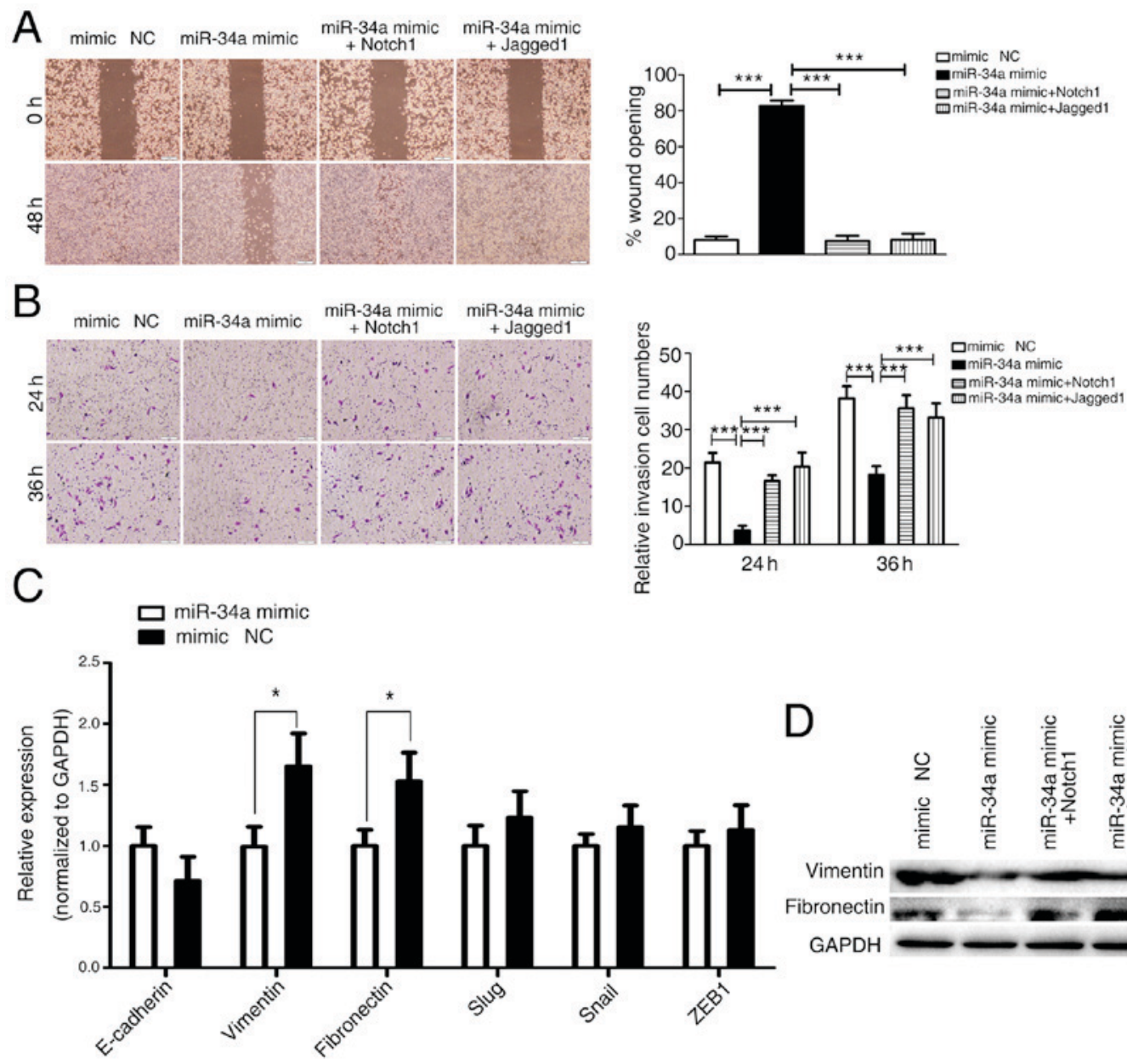

Figure 3. miR-34a inhibits tumor cell migration and invasion by targeting Notch1 and Jagged1. (A) SW480 cells were transfected with miR-34a (or mimic $\mathrm{NC}$ ) followed by Notch1 or Jagged 1 transfection. Cells were subjected to the wound closure assay in a time-dependent manner. Images captured at 0 and $48 \mathrm{~h}$ are provided. Representative images of the migrated stained cells are also provided (left). Cells in 3 randomly selected areas were counted and statistical analyses were performed. The data are expressed as the mean $\pm \mathrm{SD}(\mathrm{n}=3)$ (right). ${ }^{*} \mathrm{P}<0.05,{ }^{* * *} \mathrm{P}<0.01$ and ${ }^{* * * *} \mathrm{P}<0.001$ vs. miR-34a mimic. (B) Transwell migration assays examining the effects of miR-34a on cell invasion ability. SW480 cells were transfected with miR-34a (or mimic NC) followed by Notch1 or Jagged1 transfection. The Transwell migration assay was performed 24 and $36 \mathrm{~h}$ later. Representative images of the migrated stained cells are provided (left). SW480 cells in 5 randomly selected areas were counted and statistical analyses were performed (right). The data are expressed as the mean $\pm \mathrm{SD}$. ${ }^{*} \mathrm{P}<0.05$, ${ }^{* *} \mathrm{P}<0.01$ and ${ }^{* * *} \mathrm{P}<0.001$ vs. miR-34a mimic. (C) Regulation of the expression of E-cadherin, vimentin, fibronectin, Slug, Snail and ZEB1 by miR-34a. SW480 cells were transfected with an miR-34a mimic or mimic NC for $48 \mathrm{~h}$. The expression of E-cadherin, vimentin, fibronectin, Slug, Snail and ZEB1 was analyzed using the reverse transcription-quantitative polymerase chain reaction. Values were normalized to GAPDH as an internal control. The data are expressed as the mean $\pm \mathrm{SD}\left(\mathrm{n}=3\right.$ ). ${ }^{*} \mathrm{P}<0.05$ vs. mimic NC. (D) SW480 cells were transfected with miR-34a (or mimic NC) followed by Notch1 or Jagged1 transfection. The protein expression of vimentin and fibronectin was assayed by western blot analysis. miR, microRNA; SD, standard deviation; E-cadherin, epithelial cadherin; Slug, zinc finger protein SNAI2; Snai1, zinc finger protein SNAI1; ZEB1, zinc finger E-box-binding homeobox 1; NC, negative control.

specifically targets Notch1 or Jagged1 to suppress CRC metastasis (Fig. 3A and B). Previous evidence suggests that the activation of Notch1 and Jagged1 induces EMT and promoted the invasion and dissemination of cancer cells (29-31). Therefore, whether miR-34a affected EMT was investigated. A panel of EMT markers, including epithelial cadherin, vimentin, fibronectin, Slug, Snail and ZEB1, were detected in SW480 cells following transfection with miR-34a mimic or mimic NC (Fig. 3C). Significant attenuation of the mRNA expression levels of two key mesenchymal markers, vimentin and fibronectin, was observed (Fig. 3C and D). In addition, the re-expression of Notch1 or Jagged1 recovered the protein expression levels of vimentin and fibronectin in miR-34a-overexpressing SW480 cells, suggesting that miR-34a suppresses CRC metastasis by targeting Notch1/Jagged1, and the downstream molecules vimentin and fibronectin.

\section{Discussion}

Tumor progression in CRC is a complex process. Metastasis is the primary characteristic of malignant tumors. It is the primary cause of mortality in the majority of patients with cancer (32). miRNAs have been described as a novel class of molecular regulators of tumorigenesis (33). Previous studies have attempted to delineate their role in the regulation of metastasis and disease progression (34-36).

MiR-34a is a member of a family of evolutionarily conserved miRNAs that are regulated by the tumor suppressor tumor protein 53 (37-39). Tazawa et al (17) have suggested that miR-34a expression levels were downregulated by $36 \%$ in CRC relative to their levels in normal tissues, although the underlying molecular mechanism for tumorigenesis remains unclear. Previously, Liu et al (40) identified that miR-34a 
inhibited prostate cancer stem cells and metastasis by directly repressing cluster of differentiation 44 expression. It was also demonstrated that miR-34a inhibits colon cancer cell migration and invasion by targeting Fra-1 (41). In addition, it was suggested that miR-34a serves a key role in tumor cell responses to chemotherapeutic agents, and may serve as a target for antitumor therapy $(10,42)$. In the present study, by assaying the expression of miR-34a in CRC cell lines and clinical specimens, it was confirmed that miR-34a was downregulated in CRC samples compared with normal tissues, and the levels of miR-34a expression were identified to be inversely associated with tumor metastasis. Additionally, it was demonstrated that increased survival was associated with high expression levels of miR-34a.

A previous study confirmed that miR-34a targeted multiple key pathways including the hepatocyte growth factor/c-Met pathway, E2F pathway and cell cycle regulator cyclin-dependent kinase 6 (17). However, the precise underlying molecular mechanism for the role of miR-34a in colorectal tumorigenesis remains unclear. Among the regulatory mechanisms targeted by miR-34a, the Notch pathway serves a prominent role in cell fate determination during cancer proliferation, apoptosis, invasion and metastasis (43). There are 4 Notch receptors (Notch1-4) and 5 Notch ligands ( $\delta$-like-1, -3 and -4, and Jagged1 and 2) in mammals. Following specific ligand binding, the intracellular part of the Notch receptor is cleaved and translocated to the nucleus, where it binds to the transcription factor recombining binding protein for immunoglobulin $\kappa \mathrm{J}$ region to activate Notch target genes $(44,45)$. Notch1 is one of the Notch receptors involved in Notch signaling, which serves a key role in the regulation of a number of fundamental cellular processes, including proliferation, stem cell maintenance, differentiation and EMT $(27,46)$. Notch1 and Jagged1 are key factors in the regulation of survival and invasion in a wide variety of cancers including gastric, salivary, pancreatic and neuroendocrine tumors, and adenoid cystic carcinoma and CRC (47-50). The luciferase activity assays of the present study demonstrated that miR-34a binds to the 3'-UTRs of Notch1 and Jagged1. Additionally, when miR-34a oligonucleotides were transfected into CRC cells, an inverse expression pattern was observed between miR-34a and Notch1 and Jagged 1 at the gene and protein levels, implying that miR-34a targets Notch1 and Jagged1 through translational arrest and mRNA degradation. Thus, it was concluded that miR-34a directly targets Notch1 and Jagged1 in CRC cells.

Considering that miR-34a and Notch1/Jagged1 are closely associated with tumor invasion and metastasis, the effects of miR-34a on these phenotypes in CRC cells were also investigated. miR-34a overexpression in SW480 cells markedly attenuated the cell migratory and invasive abilities, whereas the overexpression of Notch1 or Jagged1 rescued the miR-34a inhibition of cell migration and invasion caused by miR-34a, suggesting that miR-34a regulation of CRC invasion and metastasis is a consequence of targeting upstream Notch signaling. In addition, an overexpression of miR-34a decreased the expression of mesenchymal markers, vimentin and fibronectin in colon cancer cells. The expression of Notch1 or Jagged1 in SW480 cells recovered the protein expression of vimentin and fibronectin by miR-34a. It is possible that miR-34a suppresses
CRC metastasis by targeting Notch1/Jagged1 and the vimentin and fibronectin downstream.

In conclusion, the results of present study support the miR-34a-mediated suppression of metastasis in CRC by targeting Notch1/Jagged1 and their downstream molecules vimentin and fibronectin. However, additional studies including in vivo assays are needed to confirm the understanding of miR-34a regulation in CRC tumorigenesis and metastasis. In addition, due to the multiple gene targets involved, miR-34a regulation of Notch signaling and the associated pathways require comprehensive investigation.

\section{Acknowledgements}

The present study was supported by the National Natural Science Foundation of China (grant no. 81472286).

\section{References}

1. Siegel RL, Miller KD and Jemal A: Cancer statistics. 2016. CA Cancer J Clin 66: 7-30, 2016.

2. Siegel R, Naishadham D and Jemal A: Cancer statistics, 2012. CA Cancer J Clin 62: 10-29, 2012.

3. Van Cutsem E, Nordlinger B, Adam R, Köhne CH, Pozzo C, Poston G, Ychou M and Rougier P; European Colorectal Metastases Treatment Group: Towards a pan-European consensus on the treatment of patients with colorectal liver metastases. Eur J Cancer 42: 2212-2221, 2006.

4. Davies RJ, Miller R and Coleman N: Colorectal cancer screening: Prospects for molecular stool analysis. Nat Rev Cancer 5: 199-209, 2005.

5. Ambros V: The functions of animal microRNAs. Nature 431: 350-355, 2004.

6. Schee K, Boye K, Abrahamsen TW, Fodstad $\varnothing$ and Flatmark K: Clinical relevance of microRNA miR-21, miR-31, miR-92a, miR-101, miR-106a and miR-145 in colorectal cancer. BMC Cancer 12: 505, 2012.

7. Wang R, Ma J, Wu Q, Xia J, Miele L, Sarkar FH and Wang Z: Functional role of miR-34 family in human cancer. Curr Drug Targets 14: 1185-1191, 2013.

8. Agostini M and Knight RA: miR-34: From bench to bedside. Oncotarget 5: 872-881, 2014.

9. Wiggins JF, Ruffino L, Kelnar K, Omotola M, Patrawala L, Brown D and Bader AG: Development of a lung cancer therapeutic based on the tumor suppressor microRNA-34. Cancer Res 70: 5923-5930, 2010.

10. Di Martino MT, Leone E, Amodio N, Foresta U, Lionetti M, Pitari MR, Cantafio ME, Gullà A, Conforti F, Morelli E, et al: Synthetic miR-34a mimics as a novel therapeutic agent for multiple myeloma: In vitro and in vivo evidence. Clin Cancer Res 18: 6260-6270, 2012.

11. Welch C, Chen Y and Stallings RL: MicroRNA-34a functions as a potential tumor suppressor by inducing apoptosis in neuroblastoma cells. Oncogene 26: 5017-5022, 2007.

12. Cole KA, Attiyeh EF, Mosse YP, Laquaglia MJ, Diskin SJ, Brodeur GM and Maris JM: A functional screen identifies miR-34a as a candidate neuroblastoma tumor suppressor gene. Mol Cancer Res 6: 735-742, 2008.

13. Li Y, Guessous F, Zhang Y, Dipierro C, Kefas B, Johnson E, Marcinkiewicz L, Jiang J, Yang Y, Schmittgen TD, et al: MicroRNA-34a inhibits glioblastoma growth by targeting multiple oncogenes. Cancer Res 69: 7569-7576, 2009.

14. Li WB, Ma MW, Dong LJ, Wang F, Chen LX and Li XR: MicroRNA-34a targets notch1 and inhibits cell proliferation in glioblastoma multiforme. Cancer Biol Ther 12: 477-483, 2011.

15. Pang RT, Leung CO, Ye TM, Liu W, Chiu PC, Lam KK, Lee KF and Yeung WS: MicroRNA-34a suppresses invasion through downregulation of Notch1 and Jagged1 in cervical carcinoma and choriocarcinoma cells. Carcinogenesis 31: 1037-1044, 2010.

16. Yang S, Li Y, Gao J, Zhang T, Li S, Luo A, Chen H, Ding F, Wang X and Liu Z: MicroRNA-34 suppresses breast cancer invasion and metastasis by directly targeting Fra-1. Oncogene 32: 4294-4303, 2013. 
17. Tazawa $\mathrm{H}$, Tsuchiya $\mathrm{N}$, Izumiya $\mathrm{M}$ and Nakagama $\mathrm{H}$ Tumor-suppressive miR-34a induces senescence-like growth arrest through modulation of the E2F pathway in human colon cancer cells. Proc Natl Acad Sci USA 104: 15472-15477, 2007.

18. Lefort K, Brooks Y, Ostano P, Cario-André M, Calpini V, Guinea-Viniegra J, Albinger-Hegyi A, Hoetzenecker W, Kolfschoten I, Wagner EF, et al: A miR-34a-SIRT6 axis in the squamous cell differentiation network. EMBO J 32: 2248-2263, 2013.

19. Rokavec M, Öner MG, Li H, Jackstadt R, Jiang L, Lodygin D, Kaller M, Horst D, Ziegler PK, Schwitalla S, et al: IL-6R/STAT3/miR-34a feedback loop promotes EMT-mediated colorectal cancer invasion and metastasis. J Clin Invest 124 $1853-1867,2014$

20. Hahn S, Jackstadt R, Siemens H, Hünten $S$ and Hermeking $H$ : SNAIL and miR-34a feed-forward regulation of ZNF281/ZBP99 promotes epithelial-mesenchymal transition. EMBO J 32: 3079-3095, 2013.

21. Nalls D, Tang SN, Rodova M, Srivastava RK and Shankar S: Targeting epigenetic regulation of miR-34a for treatment of pancreatic cancer by inhibition of pancreatic cancer stem cells. PLoS One 6: e24099, 2011.

22. Bu P, Chen KY, Chen JH, Wang L, Walters J, Shin YJ, Goerger JP, Sun J, Witherspoon M, Rakhilin N, et al: A microRNA miR-34a-regulated bimodal switch targets Notch in colon cancer stem cells. Cell Stem Cell 12: 602-615, 2013

23. Cheng CY, Hwang CI, Corney DC, Flesken-Nikitin A, Jiang L, Oner GM, Munroe RJ, Schimenti JC, Hermeking $\mathrm{H}$ and Nikitin AY: miR-34 cooperates with p53 in suppression of prostate cancer by joint regulation of stem cell compartment. Cell Rep 6: 1000-1007, 2014.

24. Liu C and Tang DG: MicroRNA regulation of cancer stem cells. Cancer Res 71: 5950-5954, 2011.

25. Wittekind C: 2010 TNM system: On the 7th edition of TNM classification of malignant tumors. Pathologe 31: 331-332, 2010 (In German)

26. Livak KJ and Schmittgen TD: Analysis of relative gene expression data using real-time quantitative PCR and the 2(-Delta Delta C(T)) method. Methods 25: 402-408, 2001.

27. Leong KG and Karsan A: Recent insights into the role of Notch signaling in tumorigenesis. Blood 107: 2223-2233, 2006.

28. Zagouras P, Stifani S, Blaumueller CM, Carcangiu ML and Artavanis-Tsakonas S: Alterations in Notch signaling in neoplastic lesions of the human cervix. Proc Natl Acad Sci USA 92: 6414-6418, 1995.

29. Du R, Sun W, Xia L, Zhao A, Yu Y, Zhao L, Wang H, Huang C and Sun S: Hypoxia-induced down-regulation of microRNA-34a promotes EMT by targeting the Notch signaling pathway in tubular epithelial cells. PLoS One 7: e30771, 2012.

30. Wang Y, Wu B, Chamberlain AA, Lui W, Koirala P, Susztak K, Klein D, Taylor V and Zhou B: Endocardial to myocardial notch-wnt-bmp axis regulates early heart valve development. PLoS One 8: e60244, 2013.

31. Zavadil J, Cermak L, Soto-Nieves $N$ and Böttinger EP: Integration of TGF-beta/Smad and Jagged1/Notch signalling in epithelial-to-mesenchymal transition. EMBO J 23: 1155-1165, 2004.

32. Hanahan D and Weinberg RA: Hallmarks of cancer: The next generation. Cell 144: 646-674, 2011.

33. Di Leva G, Garofalo M and Croce CM: MicroRNAs in cancer. Annu Rev Pathol 9: 287-314, 2014.

34. Asangani IA, Rasheed SA, Nikolova DA, Leupold JH, Colburn NH, Post S and Allgayer H: MicroRNA-21 (miR-21) post-transcriptionally downregulates tumor suppressor Pded 4 and stimulates invasion, intravasation and metastasis in colorectal cancer. Oncogene 27: 2128-2136, 2008.
35. Ma L, Teruya-Feldstein $\mathrm{J}$ and Weinberg RA: Tumour invasion and metastasis initiated by microRNA-10b in breast cancer. Nature 449: 682-688, 2007.

36. Valastyan S, Reinhardt F, Benaich N, Calogrias D, Szász AM, Wang ZC, Brock JE, Richardson AL and Weinberg RA: A pleiotropically acting microRNA, miR-31, inhibits breast cancer metastasis. Cell 137: 1032-1046, 2009.

37. Hermeking H: The miR-34 family in cancer and apoptosis. Cell Death Differ 17: 193-199, 2010.

38. Hermeking H: p53 enters the microRNA world. Cancer Cell 12: 414-418, 2007.

39. Chang TC, Wentzel EA, Kent OA, Ramachandran K, Mullendore M, Lee KH, Feldmann G, Yamakuchi M, Ferlito M, Lowenstein CJ, et al: Transactivation of miR-34a by p53 broadly influences gene expression and promotes apoptosis. Mol Cell 26: 745-752, 2007.

40. Liu C, Kelnar K, Liu B, Chen X, Calhoun-Davis T, Li H, Patrawala L, Yan H, Jeter C, Honorio S, et al: The microRNA miR-34a inhibits prostate cancer stem cells and metastasis by directly repressing CD44. Nat Med 2: 211-215, 2011.

41. Wu J, Wu G, Lv L, Ren YF, Zhang XJ, Xue YF, Li G, Lu X, Sun Z and Tang KF: MicroRNA-34a inhibits migration and invasion of colon cancer cells via targeting to Fra-1. Carcinogenesis 33: 519-528, 2012.

42. Li XJ, Ren ZJ and Tang JH: MicroRNA-34a: A potential therapeutic target in human cancer. Cell Death Dis 5: e1327, 2014.

43. Ntziachristos P, Lim JS, Sage J and Aifantis I: From fly wings to targeted cancer therapies: A centennial for notch signaling. Cancer Cell 25: 318-334, 2014.

44. Artavanis-Tsakonas S, Rand MD and Lake RJ: Notch signaling: Cell fate control and signal integration in development. Science 284: 770-776, 1999.

45. Kopan R and Ilagan MX: The canonical Notch signaling pathway: Unfolding the activation mechanism. Cell 137: 216-233, 2009.

46. Dotto GP: Notch tumor suppressor function. Oncogene 27: 5115-5123, 2008.

47. Su BH, Qu J, Song M, Huang XY, Hu XM, Xie J, Zhao Y, Ding LC, She L, Chen J, et al: NOTCH1 signaling contributes to cell growth, anti-apoptosis and metastasis in salivary adenoid cystic carcinoma. Oncotarget 5: 6885-6895, 2014.

48. Zhang L, Dong Y, Zhu N, Tsoi H, Zhao Z, Wu CW, Wang K, Zheng S, Ng SS, Chan FK, et al: microRNA-139-5p exerts tumor suppressor function by targeting NOTCH1 in colorectal cancer. Mol Cancer 13: 124, 2014.

49. Zhang H, Wang X, Xu J and Sun Y: Notch1 activation is a poor prognostic factor in patients with gastric cancer. Br J Cancer 110: 2283-2290, 2014

50. Zhang J, Francois R, Iyer R, Seshadri M, Zajac-Kaye M and Hochwald SN: Current understanding of the molecular biology of pancreatic neuroendocrine tumors. J Natl Cancer Inst 105: 1005-1017, 2013 